\title{
Note on an equation occurring in a problem of Nicole Brillouët-Belluot
}

\author{
Witold JARCZYK AND JANusZ MORAWIEC
}

\begin{abstract}
We study the functional equation $f(x) f^{-1}(x)=x^{2}$ imposing no continuity assumptions on its bijective solutions defined on an interval. All the continuous bijections satisfying the equation were determined by the second author in (Aequat. Math. (in print), 2011) when solving the problem (Problem posed during the forty-ninth International Symposium on Functional Equations 2011) posed by Brillouët-Belluot.
\end{abstract}

Mathematics Subject Classification (2010). Primary 39B22; Secondary 39B12, 26A18.

Keywords. Iterative functional equation, bijection, iterate, general solution.

\section{Introduction}

In [2] the second author solved the problem posed in [1] proving that any continuous bijection of an arbitrary real interval $I$, satisfying the equation

$$
f(x) f^{-1}(x)=x^{2},
$$

is either linear:

$$
f(x)=c x, \quad x \in I,
$$

or of the form

$$
f(x)= \begin{cases}a x, & \text { if } x \in I \cap(-\infty, 0) \\ b x, & \text { if } x \in I \cap[0, \infty)\end{cases}
$$

The following two examples show that the continuity of a solution cannot be omitted there.

Example 1. The formula

$$
f(x)= \begin{cases}x, & \text { if } x \in 4^{n}\left(\frac{1}{2}, 1\right] \text { and } \quad n \in \mathbb{Z} \\ 4 x, & \text { if } x \in 4^{n}(1,2] \text { and } \quad n \in \mathbb{Z}\end{cases}
$$


provides a bijective solution $f:(0, \infty) \rightarrow(0, \infty)$ of $(1.1)$ which is discontinuous exactly at the powers of 2 .

Example 2. Let $I$ be an interval and let $A$ be its symmetric subset such that both $A$ and $I \backslash A$ contain a non-zero number. The function $f: I \rightarrow I$, given by

$$
f(x)= \begin{cases}-x, & \text { if } x \in A, \\ x, & \text { if } x \in I \backslash A,\end{cases}
$$

is a discontinuous bijection satisfying (1.1).

Note that there are bijective solutions of (1.1) that are discontinuous at exactly one point; to see this take $I=(-a, a]$ with $a>0$ and set $A=(-a, a)$ in Example 2.

In what follows $f$ stands for a bijection of an interval $I$, satisfying (1.1), and $f^{n}$, where $n \in \mathbb{Z}$, denotes the $n$-th iterate of $f$. The only exception is Theorem 2.4, where $I$ is replaced by an arbitrary set $X$ of reals. (Note, however, that also some of the remaining results can be easily proved for bijections of $X$, with no essential changes in the proofs.)

Remark 1.1. (i) [2, Lemma 2.1] If $0 \in I$ then $f(0)=0$.

(ii) $[2$, Lemma 2.4] The function $g:-I \rightarrow-I$, given by $g(x)=-f(-x)$, is a bijective solution of (1.1).

\section{Main results}

We start with the following result where no continuity assumption is imposed on a solution of (1.1).

Theorem 2.1. (i) If $I \subset[0, \infty)$ or $I \subset(-\infty, 0]$, and $I$ is not a half-line ending with 0 , then

$$
f(x)=x, \quad x \in I .
$$

(ii) If I is bounded then

$$
|f(x)|=|x|, \quad x \in I .
$$

Remark 2.2. It is an easy observation that, because of (1.1), for every $x \in I$ we have $|f(x)|=|x|$ if and only if $f^{2}(x)=x$.

In the proof of Theorem 2.1 the following lemma plays a crucial role. Its first assertion is, in fact, Lemma 2.2 from [2] and the second one follows immediately.

Lemma 2.3. For every $x \in I \backslash\{0\}$ the sequence $\left(f^{n}(x)\right)_{n \in \mathbb{Z}}$ is geometric:

$$
f^{n}(x)=\left(\frac{f(x)}{x}\right)^{n} x, \quad n \in \mathbb{Z} .
$$


In particular,

if $0<|f(x)|<|x|, \quad$ then $\lim _{n \rightarrow+\infty} f^{n}(x)=0$ and $\lim _{n \rightarrow-\infty}\left|f^{n}(x)\right|=+\infty$ and

if $|f(x)|>|x|>0$, then $\lim _{n \rightarrow+\infty}\left|f^{n}(x)\right|=+\infty$ and $\lim _{n \rightarrow-\infty} f^{n}(x)=0$ for any $x \in I$.

Proof of Theorem 2.1. (i) By Remark 1.1 we may assume that $I \subset(0, \infty)$. Then, as $I \neq(0, \infty)$ it follows from Lemma 2.3 that $f(x)=x$ for all $x \in I$.

(ii) Since the endpoints of $I$ are finite, Lemma 2.3 gives the assertion immediately.

As it follows from Theorem 2.1(i) we may expect discontinuous bijective solutions of (1.1) defined on $I$ only if either $I$ is a half-line ending with 0 , or $0 \in \operatorname{int} I$. Example 1 together with Remark 1.1(ii) as well as Example 2 show that this is actually the case.

Example 1 also suggested the following description of the general solution of (1.1) in the class of bijections of an arbitrary set $X \subset \mathbb{R}$.

Theorem 2.4. Let $X$ be a set of reals. Equation (1.1) has a solution which is a bijection of $X$ if and only if there is a partition of $X$ into pairwise disjoint sets of elements of some two-sided geometric sequences. If $\left\{A_{t}\right\}_{t \in T}$ is such a partition of $X$, viz.

$$
A_{t}=\left\{a_{t} q_{t}^{n}: n \in \mathbb{Z}\right\}, \quad t \in T,
$$

with some $a_{t} \in X$ and $q_{t} \in \mathbb{R} \backslash\{0\}$, then the formula

$$
f\left(a_{t} q_{t}^{n}\right)=a_{t} q_{t}^{n+1}
$$

defines a bijection of $X$ which satisfies (1.1). Conversely: any bijection of $X$ satisfying (1.1) can be obtained in such a manner.

Proof. To obtain the first part of the theorem it is enough to take any $x \in X$ of the form $a_{t} q_{t}^{n}$ and observe that

$$
f(x) f^{-1}(x)=f\left(a_{t} q_{t}^{n}\right) f^{-1}\left(a_{t} q_{t}^{n}\right)=a_{t} q_{t}^{n+1} a_{t} q_{t}^{n-1}=\left(a_{t} q_{t}^{n}\right)^{2}=x^{2} .
$$

Conversely, if $f$ is a bijection of $X$ satisfying (1.1), then it is sufficient to consider the partition of $X$ into the orbits $\left\{f^{n}(x): n \in \mathbb{Z}\right\}$ of all elements $x$ of $X$; it follows immediately from (1.1) that all the sequences $\left(f^{n}(x)\right)_{n \in \mathbb{Z}}$ are geometric (in the case $X=I$ it is explicitly indicated in Lemma 2.3).

It turns out that if $0 \in \operatorname{int} I$ and $I$ is bounded, then Example 2 yields a form of general solution of (1.1) which can be seen from the next result. 
Theorem 2.5. Assume that $0 \in \operatorname{int} I$ and $I$ is bounded. Then there is a non-void symmetric set $A \subset I$ such that (1.4) holds.

Proof. The assertion can be easily deduced from Theorem 2.4 and Lemma 2.3. Nevertheless we present another argument.

By Theorem 2.1(ii) we know that $f(x) \in\{-x, x\}$ for every $x \in I$, and thus, as $f$ is a bijection, $f(x)=x$ for all $x \in I \backslash(-I)$. Put $A=\{x \in I: f(x)=-x\}$. Then $0 \in A \subset I \cap(-I)$. Clearly (1.4) holds true. If $x \in A \backslash\{0\}$ then $f(x)=-x$, whence, by (1.1), $x f(-x)=x f^{2}(x)=f(x)^{2}=(-x)^{2}$, i.e. $f(-x)=-(-x)$ and $-x \in A$. Consequently, $A$ is symmetric.

Examples 1 and 2 show that when $I$ is a half-line ending with 0 or $0 \in \operatorname{int} I$, it is difficult to find conditions which guarantee that a solution of (1.1) is of one of the forms in (1.2) and (1.3), other than its continuity. We complete the note with two possible trials into this direction.

Theorem 2.6. Assume that $I$ is a half-line ending with 0 and the function $x \mapsto f(x) / x$ has a (finite or not) limit $c$ at at least one endpoint of $I$. Then $c$ is finite and positive, and (1.2) holds.

Proof. Because of Remark 1.1 we may assume that $I=(0, \infty)$.

Define $g: I \rightarrow I$ by

$$
g(x)=\frac{f(x)}{x} .
$$

It follows from Lemma 2.3 that

$$
\frac{f^{n+1}(x)}{f^{n}(x)}=\frac{f(x)}{x}, \quad x \in I, n \in \mathbb{Z},
$$

that is

$$
g\left(f^{n}(x)\right)=g(x), \quad x \in I, n \in \mathbb{Z} .
$$

Put $C=\{x \in I: f(x) \neq x\}$. Without loss of generality we may assume that $C \neq \emptyset$. From (2.3) and the second part of Lemma 2.3 we deduce that $g(x)=c$, i.e. $f(x)=c x$ for all $x \in C$. Consequently, $c \in(0, \infty) \backslash\{1\}$ because $C \neq \emptyset$, and $C=\{x \in I: f(x)=c x\}$. Moreover, as

$$
I=f(I)=f(C) \cup f(I \backslash C)=c C \cup(I \backslash C) \text { and } f(C) \cap f(I \backslash C)=\emptyset,
$$

it follows that $c C=C$.

Since $c$ is the limit of $g$ at an endpoint of $I$ and $g$ takes at most two values: $c$ and 1 , there is a vicinity $U$ of the endpoint such that $U \subset C$. Take any $x \in I$ and $n \in \mathbb{Z}$ such that $c^{n} x \in U$. Then $x \in c^{-n} U \subset c^{-n} C=C$. This shows that $C=I$ and this completes the proof.

A counterpart of Theorem 2.6 for intervals containing 0 in their interior reads as follows. 
Theorem 2.7. Assume that $0 \in \operatorname{int} I$ and the function $x \mapsto f(x) / x$ has (finite or not) left hand limit $a$ and right hand limit $b$ at 0 . Then $a, b$ are finite and non-zero, and either both are positive, or $a=b$. Moreover, either (1.3), or (2.1) hold.

Proof. We argue similarly as in the proof of Theorem 2.6. Defining $g: I \backslash\{0\} \rightarrow$ $I \backslash\{0\}$ by $(2.2)$ we come to

$$
g\left(f^{2 n-1}(x)\right)=g(x)=g\left(f^{2 n}(x)\right), \quad x \in I \backslash\{0\}, n \in \mathbb{Z} .
$$

At first we claim that $a, b \in \mathbb{R} \backslash\{0\}$ and

$$
\begin{array}{ll}
\text { either } f(x)=a x, \text { or }|f(x)|=|x|, & x \in I \cap(-\infty, 0], \\
\text { either } f(x)=b x, \text { or }|f(x)|=|x|, & x \in I \cap[0, \infty) .
\end{array}
$$

In fact, if $|f(x)|=|x|$ for all $x \in I \cap(-\infty, 0)$, then $|a|=1$. Otherwise take any $x \in I \cap(-\infty, 0)$ with $|f(x)| \neq|x|$. Then the second part of Lemma 2.3 and (2.4) implies $g(x)=a$, whence $a \in \mathbb{R} \backslash\{0\}$ and $f(x)=a x$, which gives (2.5a). Similarly one can get $(2.5 \mathrm{~b})$.

Now we prove that either $a, b$ are positive, or $a=b$. Assume, for instance, that $a<0$. If $a=-1$ then, by (2.5a), the function $g$ takes at most two values in $I \cap(-\infty, 0)$, viz. -1 and 1 , and thus $f(x)=-x$ in a left vicinity $U$ of 0 . Now (1.1) implies $f(-x)=f(f(x))=f^{2}(x)=f(x)^{2} / x=(-x)^{2} / x=x$ for every $x \in U$, i.e. $f(x)=-x$ in $-U$, whence $b=-1$, that is $a=b$. If $a \in(-\infty, 0) \backslash\{-1\}$ then we can find an $x \in I \backslash\{0\}$ such that $f(x) / x \in$ $(-\infty, 0) \backslash\{-1\}$, and thus, because of Lemma 2.3, we have $f^{2 n}(x) / f^{2 n-1}(x)<0$ for every $n \in \mathbb{Z}$. Consequently, (2.4) forces $a=b$ in that case as well. Similarly we prove that $a=b$ whenever $b<0$.

We show that

$$
\begin{array}{ll}
\text { either } f^{2}(x)=a^{2} x, \text { or } f^{2}(x)=x, & x \in I \cap(-\infty, 0], \\
\text { either } f^{2}(x)=b^{2} x, \text { or } f^{2}(x)=x, & x \in I \cap[0, \infty) .
\end{array}
$$

Take any $x \in I \cap(-\infty, 0)$. If $|f(x)|=|x|$ then, by Remark 2.2, we have $f^{2}(x)=x$. So, because of (2.5a), we may assume that $f(x)=a x$. We prove that $f^{2}(x)=a^{2} x$. If $a>0$ then $a x \in I \cap(-\infty, 0)$ whence, by (2.5a), we get either $f^{2}(x)=f(a x)=a^{2} x$, or $\left|f^{2}(x)\right|=|f(a x)|=|a x|=|a||x|$, i.e. $f^{2}(x)=|a| x$, since $x f^{2}(x) \geq 0$ by (1.1). If $a<0$ then $b=a$ and $a x \in I \cap(0, \infty)$, and thus, on account of $(2.5 \mathrm{~b})$, we have either $f^{2}(x)=f(a x)=b a x=a^{2} x$, or again $f^{2}(x)=|a| x$. In both cases it remains to consider the equality $f^{2}(x)=|a| x$. Then (1.1) gives $x \cdot|a| x=(a x)^{2}$, i.e. $|a|=1$ and, consequently, we again come to $f^{2}(x)=a^{2} x$. This yields (2.6a). Similarly one can obtain (2.6b).

Now we prove that

$$
\begin{aligned}
& f^{2}(x)=a^{2} x, \quad x \in I \cap(-\infty, 0], \\
& f^{2}(x)=b^{2} x, \quad x \in I \cap[0, \infty) .
\end{aligned}
$$


Due to condition (2.6a) we may assume that $a^{2} \neq 1$. Let $A=\{x \in I \cap(-\infty, 0]$ : $\left.f^{2}(x)=a^{2} x\right\}$. Since $a$ and $b$ are finite, we have $f(0-)=f(0+)=0$, and thus

$$
\lim _{x \rightarrow 0-} \frac{f^{2}(x)}{x}=\lim _{x \rightarrow 0-} \frac{f(f(x))}{f(x)} \frac{f(x)}{x}=a^{2}
$$

(remember that $b=a$ when $a$ is negative!). Making use of (2.6a) we see that the function $I \cap(-\infty, 0) \ni x \mapsto f^{2}(x) / x$ takes at most two values: $a^{2}$ and 1 . Therefore we can find a left vicinity $U$ of 0 such that $U \subset A$. Moreover, as

$$
\begin{aligned}
I \cap(-\infty, 0] & =f^{2}(I \cap(-\infty, 0])=f^{2}(A) \cup f^{2}(I \cap(-\infty, 0] \backslash A), \\
& =a^{2} A \cup(I \cap(-\infty, 0] \backslash A)
\end{aligned}
$$

and

$$
f^{2}(A) \cap f^{2}(I \cap(-\infty, 0] \backslash A)=\emptyset,
$$

we have $a^{2} A=A$. Take any $x \in I \cap(-\infty, 0)$ and $n \in \mathbb{Z}$ such that $a^{2 n} x \in U$. Then $x \in a^{-2 n} U \subset a^{-2 n} A=A$. This shows that $A=I \cap(-\infty, 0]$, which is (2.7a). Similarly one can prove (2.7b).

We prove that

either $f(x)=a x, x \in I \cap(-\infty, 0], \quad$ or $|f(x)|=|x|, x \in I \cap(-\infty, 0]$,

$$
\text { either } f(x)=b x, x \in I \cap[0, \infty) \text {, or }|f(x)|=|x|, x \in I \cap[0, \infty) \text {. }
$$

First assume that $\left|f\left(x_{0}\right)\right|=\left|x_{0}\right|$ for an $x_{0} \in I \cap(-\infty, 0)$. Then, because of Remark 2.2, we have $f^{2}\left(x_{0}\right)=x_{0}$ which jointly with (2.7a) gives $|a|=1$. Consequently, condition (2.5a) implies $|f(x)|=|x|$ for all $x \in I \cap(-\infty, 0]$. On the other hand, if $|f(x)| \neq|x|$ for each $x \in I \cap(-\infty, 0)$, then (2.5a) yields $f(x)=a x, x \in I \cap(-\infty, 0]$. Condition $(2.8 \mathrm{~b})$ can be proved similarly.

It follows from $(2.8 \mathrm{a})$ and $(2.8 \mathrm{~b})$ that to complete the proof of the theorem it is enough to consider the following cases only:

$$
f(x)=a x, x \in I \cap(-\infty, 0], \quad \text { and } \quad|f(x)|=|x|, x \in I \cap[0, \infty),
$$

and, symmetrically,

$$
|f(x)|=|x|, x \in I \cap(-\infty, 0], \quad \text { and } \quad f(x)=b x, x \in I \cap[0, \infty) .
$$

Assume, for instance, the first possibility. If $f(x)=x, x \in I \cap[0, \infty)$, then (1.3) holds with $b=1$. Otherwise $f\left(x_{0}\right)=-x_{0}$ for an $x_{0} \in I \cap(0, \infty)$, whence $f^{2}\left(x_{0}\right)=x_{0}$ on account of Remark 2.2, and thus $x_{0}=f^{2}\left(x_{0}\right)=f\left(-x_{0}\right)=$ $a\left(-x_{0}\right)$. Therefore $a=-1$ and, consequently, (2.1) holds.

We complete the paper with an example showing that the assertion of Theorem 2.7 can not be improved. More precisely, we show that the existence of one-sided limits of the function $I \backslash\{0\} \ni x \mapsto f(x) / x$ at 0 does not imply the continuity of $f$. 
Example 3. Let $I=[-2,2]$. Putting $A=[-1,1]$ in Example 2 we come to a discontinuous solution $f: I \rightarrow I$ of $(1,1)$ such that $\lim _{x \rightarrow 0-} f(x) / x=$ $\lim _{x \rightarrow 0+} f(x) / x=-1$. Taking $A=[-2,-1] \cup[1,2]$ there, we obtain a discontinuous solution $f: I \rightarrow I$ with $\lim _{x \rightarrow 0-} f(x) / x=\lim _{x \rightarrow 0+} f(x) / x=1$.

Observe, however, that any function of the form in (2.1), having one-sided limits at 0 , is continuous in a neighbourhood of 0 . If, in addition, such an $f$ satisfies (1.1), then there is a neighbourhood $U$ of 0 such that either $f(x)=$ $x, x \in U$, or $f(x)=-x, x \in U$.

\section{Acknowledgements}

The second author was supported by Silesian University Mathematics Department (Iterative Functional Equations and Real Analysis program).

Open Access. This article is distributed under the terms of the Creative Commons Attribution Noncommercial License which permits any noncommercial use, distribution, and reproduction in any medium, provided the original author(s) and source are credited.

\section{References}

[1] Brillouët-Belluot, N.: Problem posed during the Forty-nine International Symposium on Functional Equations. Graz-Mariatrost, Austria (2011)

[2] Morawiec, J.: On a problem of Nicole Brillouët-Belluot. Aequat. Math. doi:10.1007/ s00010-011-0096-8 (2011)

Witold Jarczyk

Faculty of Mathematics, Computer Sciences and Econometrics

University of Zielona Góra

Szafrana $4 \mathrm{a}$

65-516 Zielona Góra

Poland

e-mail: w.jarczyk@wmie.uz.zgora.pl

Janusz Morawiec

Institute of Mathematics

University of Silesia

Bankowa 14

40-007 Katowice

Poland

e-mail: morawiec@math.us.edu.pl

Received: July 14, 2011

Revised: August 16, 2011 